Supplement of Clim. Past, 15, 1327-1344, 2019

https://doi.org/10.5194/cp-15-1327-2019-supplement

(C) Author(s) 2019. This work is distributed under

the Creative Commons Attribution 4.0 License.

(c) (1)

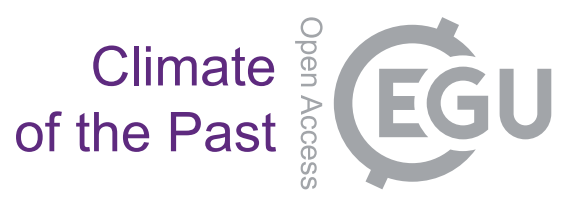

Supplement of

\title{
Central Tethyan platform-top hypoxia during Oceanic Anoxic Event 1a
}

Alexander Hueter et al.

Correspondence to: Alexander Hueter (alexander.hueter@rub.de)

The copyright of individual parts of the supplement might differ from the CC BY 4.0 License. 
Table 1. Redox sensitive trace elements arsenic, vanadium, molybdenum and uranium, as well as aluminum (ppm). Every sample was measured 6 times ( 2 spots, 3 measurements each) by LA-ICP-MS. The mean values were calculated. (This data is shown in Fig. 4).

\begin{tabular}{|c|c|c|c|c|c|}
\hline Sample No. & As mean (ppm) & $\mathrm{V}$ mean $(\mathrm{ppm})$ & Mo mean (ppm) & $\mathbf{U}$ (ppm) & $\overline{\mathrm{Al} \text { (ppm) }}$ \\
\hline KAN 8.25 & 0.730 & 7.973 & 3.485 & 1.989 & 1404.946 \\
\hline KAN 11.0 & 1.502 & 7.812 & 4.353 & 1.742 & 217.815 \\
\hline KAN 11.9 & 2.200 & 19.491 & 1.789 & 1.456 & 342.524 \\
\hline KAN 12.8 & 2.300 & NA & 7.162 & 1.660 & 295.317 \\
\hline KAN 13.2 & 3.246 & 17.604 & 4.497 & 1.162 & 818.487 \\
\hline KAN 14.2 & 0.500 & 7.856 & 0.511 & 1.223 & 150.915 \\
\hline KAN 15.4 & 0.404 & 7.327 & 0.707 & 1.408 & 141.018 \\
\hline KAN 16.3 & 0.620 & 10.911 & 0.813 & 1.141 & 504.974 \\
\hline KAN 16.6 & 1.354 & 16.061 & 2.300 & 1.274 & 1149.653 \\
\hline KAN 16.85 & 1.114 & 9.237 & 0.940 & 1.336 & 322.943 \\
\hline KAN 18.7 & 0.846 & 8.694 & 0.890 & 0.934 & 306.271 \\
\hline KAN 19.0 & 1.908 & 7.325 & 1.401 & 0.947 & 336.278 \\
\hline KAN 19.3 & 0.739 & 6.537 & 0.922 & 0.795 & 359.915 \\
\hline KAN 20.0 & 0.772 & 7.412 & 0.943 & 0.929 & 457.109 \\
\hline KAN 20.3 & 0.487 & 8.640 & 0.554 & 0.834 & 379.191 \\
\hline KAN 20.8 & 0.494 & 8.381 & 0.568 & 0.769 & 571.012 \\
\hline KAN 21.2 & 0.347 & 3.269 & 0.739 & 0.663 & 17.159 \\
\hline KAN 21.85 & 0.537 & 5.980 & 0.709 & 0.907 & 420.649 \\
\hline KAN 22.4 & 0.500 & 5.651 & 0.527 & 0.893 & 504.185 \\
\hline KAN 22.7 & 0.661 & 14.543 & 2.656 & 0.907 & 616.569 \\
\hline KAN 23.3 & 0.799 & 15.898 & 3.663 & 1.183 & 441.366 \\
\hline KAN 23.6 & 0.492 & 12.632 & 2.429 & 0.936 & 653.257 \\
\hline KAN 23.9 & 0.886 & 11.636 & 3.761 & 0.982 & 365.236 \\
\hline KAN 24.2 & 1.580 & NA & NA & 1.079 & 450.519 \\
\hline KAN 24.8 & NA & 11.120 & 0.806 & 1.156 & 403.832 \\
\hline KAN 25.2 & 0.773 & 6.725 & 1.601 & 0.762 & 363.384 \\
\hline KAN 25.5 & 1.176 & 4.734 & NA & 0.547 & 154.734 \\
\hline KAN 26.4 & 0.841 & 11.357 & 1.085 & 0.825 & 460.276 \\
\hline KAN 26.6 & 1.352 & 11.378 & 2.474 & 0.850 & 474.495 \\
\hline KAN 27.0 & 0.146 & NA & NA & 0.524 & 89.098 \\
\hline KAN 27.6 & 0.300 & 2.943 & 0.183 & 0.459 & 76.497 \\
\hline KAN 27.9 & 0.173 & 3.248 & 0.195 & 0.443 & 143.370 \\
\hline KAN 29.4 & 0.074 & NA & 0.130 & 0.467 & 33.601 \\
\hline KAN 30.8 & 0.361 & 11.558 & 1.039 & 0.600 & 476.376 \\
\hline
\end{tabular}


Table 2. Rare Earth Elements cerium, lanthanum and praseodymium (ppm) and Ce anomalies. REEs and redox sensitive trace elements were measured using LA-ICP-MS. Ce anomalies were defined following Nozaki`s calculation (2008): $\mathrm{Ce} / \mathrm{Ce}^{*}=2 \mathrm{Ce}_{\mathrm{N}} /\left(\mathrm{La}_{\mathrm{N}}+\operatorname{Pr}_{\mathrm{N}}\right)$. (This data is shown in Fig. 5).

\begin{tabular}{ccccc}
\hline Sample & Ce mean (ppm) & La mean (ppm) & Pr mean (ppm) & Ce/Ce* \\
\hline KAN 12,8 & 0.015 & 0.031 & 0.016 & 0.642 \\
KAN 13,2 & 0.013 & 0.040 & 0.019 & 0.423 \\
KAN 14,2 & 0.004 & 0.014 & 0.005 & 0.408 \\
KAN 15,4 & 0.005 & 0.017 & 0.007 & 0.400 \\
KAN 16,3 & 0.010 & 0.033 & 0.016 & 0.422 \\
KAN 16,6 & 0.012 & 0.032 & 0.016 & 0.522 \\
KAN 16,85 & 0.011 & 0.029 & 0.019 & 0.445 \\
KAN 20,8 & 0.012 & 0.023 & 0.016 & 0.588 \\
KAN 21,2 & 0.011 & 0.017 & 0.012 & 0.776 \\
KAN 21,85 & 0.008 & 0.016 & 0.010 & 0.626 \\
KAN 22,4 & 0.013 & 0.020 & 0.014 & 0.786 \\
KAN 22,7 & 0.016 & 0.020 & 0.017 & 0.876 \\
KAN 24,65 & 0.013 & 0.015 & 0.013 & 0.946 \\
KAN 24,8 & 0.020 & 0.023 & 0.020 & 0.929 \\
KAN 25,2 & 0.017 & 0.018 & 0.017 & 0.953 \\
KAN 25,5 & 0.014 & 0.018 & 0.015 & 0.868 \\
KAN 26,4 & 0.016 & 0.029 & 0.019 & 0.678 \\
KAN 26,6 & 0.014 & 0.025 & 0.014 & 0.724 \\
KAN 27,0 & 0.012 & 0.030 & 0.013 & 0.584 \\
KAN 27,6 & 0.015 & 0.048 & 0.015 & 0.471 \\
\hline
\end{tabular}


Table 3. Uranium isotope ratios and twice the standard deviation. Uranium isotope analysis were performed using MC-ICP-MS. Results are provided in the delta-notation: $\delta^{238} U$ in $\%=\left[\left({ }^{238} U /{ }^{235} U\right)_{\text {sample }} /\left({ }^{238} U /{ }^{235} U\right)_{\text {standard }}-1\right]$ $\mathrm{x}$ 1000. (This data is shown in Fig. 5).

\begin{tabular}{ccc}
\hline Sample & mean $^{238} \mathbf{U}^{235} \mathbf{U}$ & 2 S.D. \\
\hline KAN12.8 & 0.260 & 0.06 \\
KAN13.2 & 0.439 & 0.04 \\
KAN16.3 & 0.363 & 0.02 \\
KAN16.6 & 0.415 & 0.04 \\
KAN16.75 & 0.264 & 0.03 \\
KAN19 & 0.180 & 0.01 \\
KAN20.0 & 0.111 & 0.04 \\
KAN20.9 & 0.092 & 0.02 \\
KAN21.2 & 0.142 & 0.04 \\
KAN22.4 & 0.074 & 0.05 \\
KAN23.3 & 0.103 & 0.16 \\
KAN23.9 & 0.154 & 0.09 \\
KAN24.8 & 0.060 & 0.02 \\
KAN25.5 & -0.021 & 0.05 \\
KAN27.6 & 0.202 & 0.05 \\
\hline
\end{tabular}


Table 4. The Lanthanum anomaly, to test whether the Ce anomaly values are genuine or an artifact caused by elevated amounts of lanthanum. The calculation of $\operatorname{Pr} / \mathrm{Pr}^{*}$ is given by $2 \operatorname{Pr}_{\mathrm{N}} /\left(\mathrm{Ce}_{\mathrm{N}}+\mathrm{Nd}_{\mathrm{N}}\right)$. Calculation of Ce/Ce* is explained in Table 2. (This data is shown in Fig. 6).

\begin{tabular}{|c|c|c|c|c|c|c|c|c|}
\hline Sample & $\begin{array}{c}\text { La } \\
(\mathbf{p p m})\end{array}$ & $\begin{array}{c}\mathrm{Ce} \\
(\mathrm{ppm})\end{array}$ & $\begin{array}{c}\text { Pr } \\
(\mathbf{p p m})\end{array}$ & $\begin{array}{c}\text { Nd } \\
(\mathbf{p p m})\end{array}$ & $\begin{array}{c}\text { Pr* } \\
(\mathbf{p p m})\end{array}$ & $\mathbf{P r} / \mathbf{P r} *$ & $\begin{array}{c}\mathrm{Ce}^{*} \\
(\mathrm{ppm})\end{array}$ & $\mathrm{Ce} / \mathrm{Ce}^{*}$ \\
\hline KAN 7,2 & 0.010 & 0.010 & 0.010 & 0.011 & 0.021 & 0.961 & 0.020 & 1.016 \\
\hline KAN 7,6 & 0.012 & 0.013 & 0.013 & 0.014 & 0.027 & 0.983 & 0.026 & 0.997 \\
\hline KAN 8,25 & 0.013 & 0.011 & 0.012 & 0.012 & 0.023 & 1.008 & 0.025 & 0.922 \\
\hline KAN 11,0 & 0.008 & 0.003 & 0.003 & 0.003 & 0.006 & 1.005 & 0.011 & 0.526 \\
\hline KAN 11,9 & 0.008 & 0.003 & 0.003 & 0.003 & 0.007 & 0.955 & 0.011 & 0.573 \\
\hline KAN 12,8 & 0.031 & 0.015 & 0.016 & 0.016 & 0.031 & 1.051 & 0.047 & 0.642 \\
\hline KAN 13,2 & 0.040 & 0.013 & 0.019 & 0.021 & 0.034 & 1.156 & 0.059 & 0.423 \\
\hline KAN 14,2 & 0.014 & 0.004 & 0.005 & 0.005 & 0.009 & 1.046 & 0.019 & 0.408 \\
\hline KAN 15,4 & 0.017 & 0.005 & 0.007 & 0.007 & 0.012 & 1.101 & 0.024 & 0.400 \\
\hline KAN 16,3 & 0.033 & 0.010 & 0.016 & 0.017 & 0.028 & 1.141 & 0.049 & 0.422 \\
\hline KAN 16,6 & 0.032 & 0.012 & 0.016 & 0.017 & 0.029 & 1.077 & 0.048 & 0.522 \\
\hline KAN 16,75 & 0.025 & 0.010 & 0.012 & 0.014 & 0.023 & 1.056 & 0.037 & 0.515 \\
\hline KAN 16,85 & 0.029 & 0.011 & 0.019 & 0.020 & 0.031 & 1.204 & 0.048 & 0.445 \\
\hline KAN 18,7 & 0.030 & 0.009 & 0.017 & 0.019 & 0.028 & 1.168 & 0.046 & 0.401 \\
\hline KAN 19,0 & 0.044 & 0.010 & 0.045 & 0.049 & 0.059 & 1.542 & 0.089 & 0.218 \\
\hline KAN 19,3 & 0.030 & 0.011 & 0.028 & 0.031 & 0.042 & 1.316 & 0.058 & 0.364 \\
\hline KAN 20,0 & 0.037 & 0.015 & 0.035 & 0.039 & 0.054 & 1.291 & 0.072 & 0.423 \\
\hline KAN 20,3 & 0.029 & 0.013 & 0.025 & 0.028 & 0.042 & 1.199 & 0.054 & 0.495 \\
\hline KAN 20,8 & 0.023 & 0.012 & 0.016 & 0.018 & 0.030 & 1.104 & 0.039 & 0.588 \\
\hline KAN 21,2 & 0.017 & 0.011 & 0.012 & 0.013 & 0.024 & 0.967 & 0.029 & 0.776 \\
\hline KAN 21,85 & 0.016 & 0.008 & 0.010 & 0.011 & 0.026 & 1.148 & 0.026 & 0.626 \\
\hline KAN 22,4 & 0.020 & 0.013 & 0.014 & 0.015 & 0.029 & 0.974 & 0.034 & 0.786 \\
\hline KAN 22,7 & 0.020 & 0.016 & 0.017 & 0.017 & 0.034 & 0.997 & 0.037 & 0.876 \\
\hline KAN 23,3 & 0.092 & 0.017 & 0.099 & 0.105 & 0.089 & 1.595 & 0.190 & 0.178 \\
\hline KAN 23,6 & 0.085 & 0.015 & 0.075 & 0.088 & 0.103 & 1.456 & 0.160 & 0.185 \\
\hline KAN 23,9 & 0.080 & 0.014 & 0.082 & 0.092 & 0.106 & 1.555 & 0.162 & 0.173 \\
\hline KAN 24,2 & 0.054 & 0.017 & 0.036 & 0.042 & 0.044 & 1.169 & 0.090 & 0.373 \\
\hline KAN 24,65 & 0.015 & 0.013 & 0.013 & 0.014 & 0.026 & 0.961 & 0.027 & 0.946 \\
\hline KAN 24,8 & 0.023 & 0.020 & 0.020 & 0.020 & 0.024 & 0.960 & 0.043 & 0.929 \\
\hline KAN 25,2 & 0.018 & 0.017 & 0.017 & 0.018 & 0.029 & 1.006 & 0.035 & 0.953 \\
\hline KAN 25,5 & 0.018 & 0.014 & 0.015 & 0.015 & 0.026 & 1.035 & 0.033 & 0.868 \\
\hline KAN 26,4 & 0.029 & 0.016 & 0.019 & 0.021 & 0.037 & 1.039 & 0.048 & 0.678 \\
\hline KAN 26,6 & 0.025 & 0.014 & 0.014 & 0.015 & 0.029 & 0.971 & 0.039 & 0.724 \\
\hline KAN 27,0 & 0.030 & 0.012 & 0.013 & 0.014 & 0.027 & 0.978 & 0.043 & 0.584 \\
\hline KAN 27,6 & 0.048 & 0.015 & 0.015 & 0.017 & 0.032 & 0.963 & 0.063 & 0.471 \\
\hline
\end{tabular}




\begin{tabular}{lllllllll} 
KAN 27,9 & 0.065 & 0.019 & 0.019 & 0.022 & 0.041 & 0.942 & 0.084 & 0.460 \\
KAN 29,4 & 0.043 & 0.026 & 0.027 & 0.031 & 0.056 & 0.960 & 0.070 & 0.729 \\
KAN 30,0 & 0.043 & 0.027 & 0.028 & 0.031 & 0.058 & 0.957 & 0.070 & 0.758 \\
KAN 30,8 & 0.080 & 0.053 & 0.053 & 0.059 & 0.113 & 0.939 & 0.133 & 0.805 \\
KAN 33,4 & 0.107 & 0.115 & 0.118 & 0.126 & 0.241 & 0.981 & 0.225 & 1.019 \\
KAN 34,0 & 0.089 & 0.082 & 0.096 & 0.103 & 0.186 & 1.037 & 0.185 & 0.886 \\
\hline
\end{tabular}

